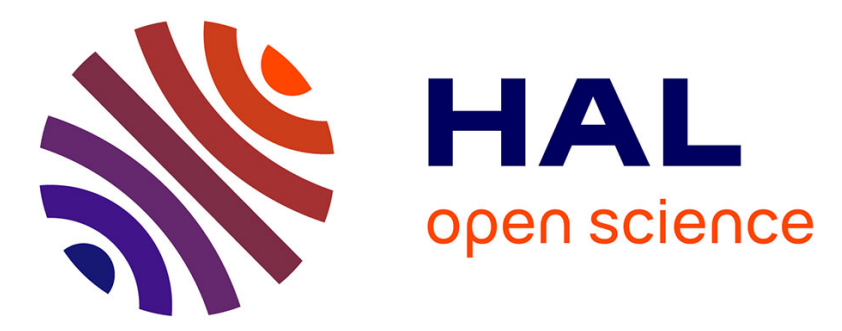

\title{
Manifestations oculaires d'une histiocytose réactionnelle systémique chez un chien Bouvier bernois
}

\author{
A. Bourguet, E. Robin, S. Chahory
}

\section{To cite this version:}

A. Bourguet, E. Robin, S. Chahory. Manifestations oculaires d'une histiocytose réactionnelle systémique chez un chien Bouvier bernois. Revue Vétérinaire Clinique, 2019, 54, pp.29 - 35. 10.1016/j.anicom.2018.10.002 . hal-03486296

\section{HAL Id: hal-03486296 https://hal.science/hal-03486296}

Submitted on 20 Dec 2021

HAL is a multi-disciplinary open access archive for the deposit and dissemination of scientific research documents, whether they are published or not. The documents may come from teaching and research institutions in France or abroad, or from public or private research centers.
L'archive ouverte pluridisciplinaire HAL, est destinée au dépôt et à la diffusion de documents scientifiques de niveau recherche, publiés ou non, émanant des établissements d'enseignement et de recherche français ou étrangers, des laboratoires publics ou privés.

\section{다)(1) $(5$}

Distributed under a Creative Commons Attribution - NonCommerciall 4.0 International 


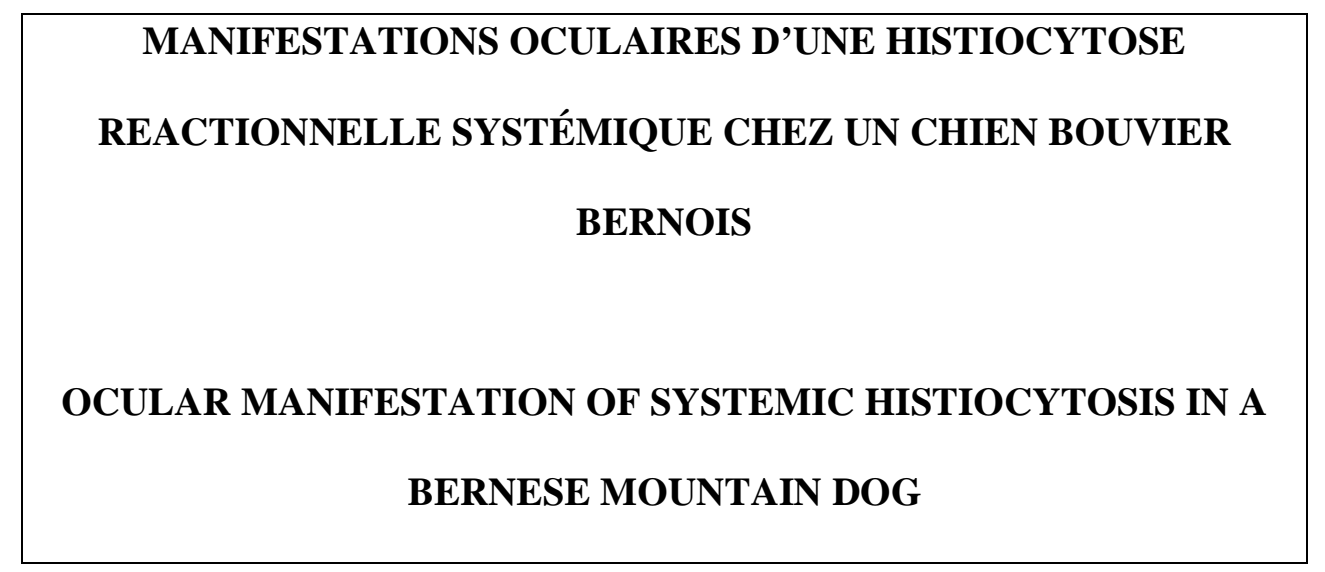

A. Bourguet*II, E. Robin††, et S. Chahory*

*Unité d'Ophtalmologie, †Unité de médecine interne, Ecole Nationale Vétérinaire d'Alfort, Université Paris - Est, Maisons-Alfort, F-94700, France; II Adresse actuelle: Centre Hospitalier Vétérinaire Atlantia, 44200 Nantes, France, $\ddagger$ Adresse actuelle: Centre Hospitalier Vétérinaire Frégis, 94110 Arcueil, France

Email : bourguetaurelie@ gmail.com

Téléphone : +33240474009

Fax : +33240892132

Adresse : Centre Hospitalier Vétérinaire Atlantia, 22 rue René Viviani, 44200 Nantes, France

\section{$\underline{\text { Remerciements }}$}

Les auteurs remercient le service d'anatomie pathologique de l'Ecole Nationale Vétérinaire d'Alfort pour la réalisation de l'interprétation des coupes histologiques. 
MANIFESTATIONS OCULAIRES D'UNE HISTIOCYTOSE REACTIONNELLE SYSTÉMIQUE CHEZ UN CHIEN BOUVIER BERNOIS

\section{OCULAR MANIFESTATIONS OF SYSTEMIC HISTIOCYTOSIS IN A}

\section{BERNESE MOUNTAIN DOG}

\section{Résumé}

Un chien, femelle, de race Bouvier bernois, est présentéen consultation pour une épisclérite aigue bilatérale associée à de multiples masses cutanées ainsi qu'une atteinte des voies respiratoires supérieures. Une histiocytose réactionnelle systémique est suspectée sur la base des signes ophtalmologiques, dermatologiques et respiratoires. L'analyse histopathologique des biopsies cutanées et épisclérales confirme le diagnostic clinique. Un traitement médical immunosuppresseur à base de corticoïdes par voie systémique et par voie topique et d'azathioprine est instauré et permet une réduction nette de la sévérité des signes cliniques. La chienne décède cependant brutalement après un épisode de dyspnée sévère 36 jours après la consultation initiale. L’intérêt de ce cas est de décrire les lésions oculaires associées à une histiocytose réactionnelle systémique chez le chien, la démarche diagnostique, le traitement et la réponse thérapeutique. La description de ce cas est intéressante du fait de la faible prévalence de cette affection et de la complexité des maladies histiocytaires canines.

\section{$\underline{\text { Abstract }}$}

A 1.5-year-old female Bernese Mountain dog was presentedfora bilateral acute episcleritisassociated with cutaneous nodules and upper respiratory tract signs. 
The diagnosis of systemic reactive histiocytosiswas based on clinical findings and confirmed by histopathological analysis of cutaneous and episcleral biopsy samples. Treatment with corticosteroids (systemically and topically) and azathioprine were effective in reducing the severity of clinical signs, however the dog died suddenly secondary to anacute respiratory distress 36 days after the initial presentation. This report describes the clinical presentation, diagnosis, histopathology and medical management ofocular manifestationssecondary to systemic reactive histiocytosis in a dog. This ocular presentation is unfrequently reported in dogs and underlines the complexy of canine histiocytic diseases.

\section{$\underline{\text { Mots clés }}$}

Azathioprine, Bouvier bernois, Episclérite, Histiocytose réactionnelle systémique, Eil

\section{Keywords}

Azathioprine, Bernese Mountain Dog, Episcleritis, Eye, Systemic reactive histiocytosis

\section{$\underline{\text { Introduction }}$}

Les proliférations histiocytaires canines constituent un groupe hétérogène d'affections incluant l'histiocytose cutanée et systémique, ainsi quedes formes tumorales telles que l'histiocytome cutanéet le sarcome histiocytaire localisé ou disséminé (histiocytose maligne) [1,2]. Une certaine confusion peut exister quant au diagnostic et au pronostic associé à chacune de ces formes de maladie histiocytaire. L'histiocytose réactionnelle systémique a été décrite récemmentet 
peut découler de l'évolution de la forme cutanée, ou d'une manifestation spontanée d'emblée généralisée. Il s'agirait d'une prolifération cellulaire résultant d'un désordre dans la régulation immunitaire après stimulation antigénique (absence de régulation négative de la réaction lymphocytaire $\mathrm{T}$ ) conduisant à l'accumulation de cellules dendritiques interstitielles activées. La pathogénie exacte n'est cependant pas totalement élucidée[1,2].

Le diagnostic définitif de l'histiocytose réactionnelle systémique se fonde sur la présentation clinique, l'analyse histopathologiqueet l'exclusion de causes infectieuses (leishmaniose notamment). Un diagnostic immunohistochimiqueest souvent nécessaire. Parmi ces proliférations, l'histiocytose réactionnelle est particulièrement fréquente chez le Bouvier bernois et se caractérise par une atteinte cutanée éventuellement associée à une atteinte oculaire et respiratoire haute[3]. Peu de données existent quant à la réponse de ces chiens au traitement immunosuppresseur, notamment lors d'atteinte de la sphère oculaire[3-5].

L'objectif de ce cas clinique est de décrire la présentation clinique, le diagnostic, la gestion thérapeutique et le pronostic d'une histiocytose réactionnelle systémique avec répercussion oculaire chez un Bouvier Bernois.

\section{Observation}

\section{Anamnèse}

Un chien Bouvier bernois, femelle non stérilisée, âgé de 1,5 ans est présenté pour l'apparition subaiguë d'une opacité cornéenne bilatérale évoluant depuis 10 jours à droite et affectant l'œil gauche depuis 2 jours. L'apparition de 
nodules cutanés sur le chanfrein et de difficultés respiratoires est également rapportée de façon concomitante aux lésions oculaires.

\section{Examen clinique général}

L'examen clinique général révèle un score de condition corporel de 4/9, une tachypnée (80 mpm), associée à un stertor. La fréquence cardiaque estde 110 battements par minute et la température corporelle de $38,2^{\circ} \mathrm{C}$. On notela présence de plaques et denodules sous-cutanés érythémateux, indurés, de 3 à 4 cm de diamètre, non prurigineux, non alopéciques, non douloureux, localisés sur le chanfrein, les membres antérieurs, le dos, les flancs et l'abdomen caudal. (Fig. 1 et 2).

\section{Examen clinique ophtalmologique}

L'évaluation de la fonction visuelle par la réalisation d'un parcours d'obstacles et par la réponse de clignement à la menace ne révèle pas d'anomalie. Les réflexes photomoteurs directs et consensuels sont présents et normaux, tandis quele réflexe à l'éclair lumineux est présent sur les deux yeux. L'examen à distance montre, de façon bilatérale et symétrique, un blépharospasme modéré, une procidence de la membrane nictitante, une hyperhémie conjonctivale et une congestion des vaisseaux épiscléraux. Une infiltration sclérale ou épisclérale bilatérale confère un aspect bombé au globe oculaire, postérieurement au limbe sur toute sa circonférence. L'examen au biomicroscope(SL-15, Kowa Company, Tokyo, Japon) révèle un œdème cornéen diffus modéré de type endothélial à droite associé à une néovascularisation profonde courte et une infiltration cellulaire limbique essentiellement temporale. A l'examen de l'œil gauche, un œdème de type endothélial est également présent avec une extension et une densité moindres 
qu'à droite, localisé dans le quadrant ventral(Fig. 3). L'effet Tyndall est négatif sur les deux yeux. Le test à la fluorescéine est négatif sur les deux yeux. Le test de Schirmer est de $23 \mathrm{~mm} / \mathrm{min}$ pour l'œil droit, et de $25 \mathrm{~mm} / \mathrm{min}$ pour l'œil gauche. Le reste de l'examen oculaire ne présente pas d'anomalie. La pression intraoculaire (Tonovet ${ }^{\circledR ;}$ Tiolat, Helsinki, Finland) donne une valeur de $12 \mathrm{~mm}$ $\mathrm{Hg}$ pour l'œil droit et $15 \mathrm{~mm} \mathrm{Hg}$ pour l'œil gauche. bilatérale d'apparition aigue associé à des lésions cutanées nodulaires et une atteinte naso-pharyngée est établi. Plusieurs hypothèses diagnostiques sont émises à ce stade : un processus histiocytaire de type histiocytose réactionnelle systémique ou sarcome histiocytaire disséminé est envisagé en premier lieu compte tenu de la localisation des lésions et la race de l'animal. Une origine tumorale (lymphome T épithéliotrope, mastocytome) ou moins probablement infectieuse (leishmaniose, atteinte mycosique systémique) sont aussi considérées.

\section{Examens complémentaires}

Un bilan biochimique complet et hématologique est réalisé. L'examen biochimique ne révèle pas d'anomalie significative, alors qu'une discrète éosinophilie est mise en évidence à la numération et formule sanguine, associée à la présence d'éosinophiles activés au frottis sanguin. Une analyse d'urine est sans anomalie.Un adénogramme (cytoponction à l'aiguille du nœud lymphatique pré-scapulaire droit) indique une population cellulaire normale et l'absence d'agents infectieux, notamment d'amastigotes. Des biopsies épisclérales et cutanées sont effectuées pour l'analyse histopathologique. Après 
biopsies révèle des caractéristiques similaires entre les lésions cutanées et épisclérales : un infiltrat périvasculaire ou périannexiel, composé de cellules histiocytaires au contour flou, au cytoplasme acidophile abondant et au noyau ovoïde excentré muni d'une chromatine fine distinctement nucléolée. Des lymphocytes matures et quelques granulocytes neutrophiles y sont associés. L'infiltrat s'organise autour des vaisseaux en formant des images en « bulbe d'oignon », avec quelques images d'angio-invasion (Fig. 4). Aucun agent pathogène (amastigote) ou contingent de cellules tumorales n'est observé. Ces images lésionnelles évoquent une histiocytose réactionnelle systémique. Un bilan d'extension est réalisé : la radiographie thoracique met en évidence la présence d'une opacité broncho-interstitielle diffuse modérée, mais en l'absence de lésions nodulaires ou d'adénopathie. L'échographie abdominale ne révèle pas d'anomalie.

\section{Traitement}

Suite àla prise en charge initiale, un ulcère cornéen droit apparait après 24 heures. L'examen ophtalmologique est stable à l'exception de l'apparition d'un ulcère stromal moyen à droite avec perte de substance stromale d'environ $30 \%$ à $40 \%$ fixant la fluorescéine, associé à la présence d'une iridocyclite caractérisée par un myosis anisocorique et un effet Tyndall modérément positif (1/4 selon la classification de Kimura) (Fig. 5).Un traitement intensif est instauré : tobramycine (Tobrex ®) toutes les 2 heures le jour, toutes les 4 heures la nuit sur l'œil droit, sérum hétérologue toutes les 2 heures le jour, toutes les 4 heures la nuit sur l'œil droit, atropine $1 \%$ collyre 2 fois par jour sur l'œil droit et méloxicam (Metacam®) $0,1 \mathrm{mg} / \mathrm{kg} / \mathrm{j}$ per os.

\section{Suivi et évolution}


Des contrôles sont régulièrement effectués après la consultation initiale,

151

152

153

154

155

156

157

158

159

160

161

162

163

164

165

166

167

168

169

170

171

172

173 à J5, J14, J22 et J29.A J5, l'examen de l'œil droit révèle une infiltration

épisclérale plus marquée qu'au contrôle précédent, une accentuation de l'œdème cornéen, une ré-épithélialisation complète de l'ulcère axial et l'apparition d'un ulcère superficiel dorsal. L'examen de l'œil gauche montre également une aggravation de l'infiltration épisclérale et l'apparition d'un œdème cornéen diffus compatible avec une endothélite (Fig. 6). Le traitement médical est adapté avec l'arrêt du méloxicam, remplacé $48 \mathrm{~h}$ plus tard par de la prednisone (Cortancyl ®) $2 \mathrm{mg} / \mathrm{kg} / \mathrm{j}$ en 2 prises. Letraitement topique sur l'œil droit est poursuivi.

A J14, la propriétaire rapporte la présence de vomissements et d'une anorexie. A l'examen oculaire, les lésions précédemment décrites à droite n'ont pas évolué, le test à la fluorescéine est négatif. Cependant, l'examen de la chambre antérieure révèle une dyscorie dorsale et un bombement irien focal dorsal, évocateurs d'une uvéite antérieure, l'effet Tyndall n'est pas évaluable en raison de l'opacité cornéenne (Fig. 7). A gauche, on note l'apparition d'une infiltration cellulaire périlimbique et d'une néovascularisation cornéenne profonde. En raison de la dégradation des signes cliniques, un traitement immunosuppresseur est introduit : azathioprine (Imurel®) $2,2 \mathrm{mg} / \mathrm{kg} / \mathrm{j}$, en plus de la corticothérapie existante, couplé à un traitement gastroprotecteur et antivomitif: sucralfate (Ulcar®), cimétidine(Zitac®) $5 \mathrm{mg} / \mathrm{kg} 3$ fois par jour, maropitant (Cerenia $\left.{ }^{\circledR}\right) 2 \mathrm{mg} / \mathrm{kg} / \mathrm{j}$. Une gestion topique de l'uvéite antérieure est initiée : dexamethasone collyre (Maxidrol@) toutes les 4 heures sur les deux yeux, atropine collyre $1 \% 2$ fois par jour à droite. 

signes digestifs ont disparu, mais un jetage nasal purulent bilatéral est apparu.

176

177

178

179

180

181

182

183

184

185

186

187

188

189

190

191

192

193

194

195

196

197

198
L'examen ophtalmologique révèle à droite une régression de l'œdème cornéen dans le quadrant dorsal. La dyscorie dorsale est stable, un effet Tyndall modéré (1/4 selon la classification de Kimura) est présent. A gauche, l'œdème cornéen est moins marqué qu'au dernier contrôle. Le traitement est poursuivi avec ajout d'une antibiothérapie systémique amoxicilline - acide clavulanique (Kesium $\left.{ }^{\circledR}\right)$ $12,5 \mathrm{mg} / \mathrm{kg}, 2$ fois par jourper os.

A J29, l'examen général indique un animal vif, mais amaigri, et la persistance du jetage nasal bilatéral. En ce qui concerne les lésions cutanées, une régression de l'érythème et de l'aspect en relief des nodules est constatée. L'examen oculaire révèle une régression de l'infiltration épisclérale sur les deux yeux, une diminution de l'œdème cornéen à droite qui reste localisé uniquement en région ventrale et temporale, ainsi qu'une résolution de l'uvéite antérieure à droite. (Fig. 8 et 9). Un contrôle biochimique et de l'hémogramme révèle une augmentation majeure des paramètres hépatiques, ainsi qu'une anémie normochrome normocytaire modérée hyporégénérative, associée à une leucocytose neutrophilique et monocytaire (Tableau 1).Compte tenu de la bithérapie immunomodulatrice (corticoïdes et azathioprine), différents tests sont proposés pour évaluer la fonction hépatique et la bonne tolérance du traitement (échographie abdominale, dosages sanguins de la bilirubine etdes acides biliaires), mais déclinés par les propriétaires.Le traitement est ajusté (passage à une administration à jours alternés de l'azathioprine), et un hépatoprotecteur est ajouté à la prescription : S-adénosyl méthionine(Zentonyl®)20 mg/kg per os.Cinq semaines après la présentation 
initiale (J36), la chienne décède brutalement, suite àun épisode de dyspnée sévère ; l'autopsie est refusée par la propriétaire.

\section{$\underline{\text { Discussion }}$}

L'histiocytose réactionnelle est une prolifération cellulaire histiocytaire, secondaire à une dérégulation du système immunitaire et affectant essentiellement des animaux jeunes à jeunes adultes (moyenne d'âge de 4 ans).

Elleévolue généralement sur un mode chronique, alternant phases cliniques et périodes de rémission [2,6]. Il s'agit une affection à prédisposition familiale établie chez les Bouviers bernois, mais également chez les Retrievers (Golden et Labrador), l'Irish Wolfhound et le Rottweiler [6-8]. Elle se caractérise cliniquement par la présence de multiples plaques et nodules cutanés non prurigineux, non douloureux, éventuellement alopéciques et ulcérés, localisés principalement sur la face, le cou, le périnée, le scrotum, les flancs et les extrémités[1,7,9,10]. Moore et al. décrivent ces nodules cutanés dans 6 cas sur 6 d'histiocytose réactionnelle systémique, Paterson et al. les rapportent également dans 5 cas sur 6 dans une autre étude[3,9]. Une polyadénomégalie est également fréquemment décrite[2].

Dans sa forme systémique, une atteinte multi organique (oculaire, splénique, hépatique, pulmonaire, ou encore de la moelle osseuse ou des cavités nasales) s'associe aux signes cutanés[3,11,12]. L’infiltration des muqueuses des cavités nasales par cette prolifération cellulaire est à l'origine d'un épaississement des muqueuses nasales, d'un stertor et éventuellement d'un jetage, Moore et al. rapportent ces signes cliniques pour 3 cas sur 6 d'histiocytose réactionnelle systémique[6,10]. 

majoritairement des atteintes des surfaces oculaires et du segment antérieur avec une infiltration conjonctivale ou épisclérale associée à un oedème cornéen, et une uvéite antérieure. Occasionnellement, une exophtalmie, une uvéite postérieure associée ou non à un décollement rétinien; des cas de glaucome secondaire sont également rapportés[3-5,9,12]. Dans l'étude de Paterson et al., sur 6 cas d'histiocytose réactionnelle chez des Bouvier bernois, 5 furent présentés pour des motifs ophtalmologiques : 4 d'entre eux pour des épisclérites/sclérites et le dernier pour l'apparition d'un ulcère stromal profond dans un contexte d'œdème cornéen diffus[9]. Scherlieet al. décrivent également un cas d'histiocytose réactionnelle chez un Bouvier bernois présenté initialement pour un ulcère descemétique avec des antécédents d'épisclérite bilatérale[5]. Les lésions d'épisclérite et de sclérite ont été démontrées comme étant secondaires à une infiltration histiocytaire et lymphoplasmocytairein $s i t u[5] . L a$ pathogénie de l'apparition des ulcères stromaux reste cependant inconnue. Une décompensation endothéliale avec apparition d'une kératopathie bulleuse aigue, une origine traumatique ou encore une surinfection locale par un traitement topique anti-inflammatoire préalable pourraient être des pistes de réflexion.

Une répercussion sur l'état général est parfois notée lors d'histiocytose réactionnelle systémique avec apparition d'une anorexie et d'un amaigrissement, avec une réponse au traitement variable pouvant conduire à l'euthanasie de l'animal[3,7,9]. Dans le cas présent, les signes cliniques observés concordent avec les données citées dans la littérature, les lésions affectant essentiellement la peau, l'épisclère et les cavités nasales. 

caractérise par un infiltrat angiocentré et vaso-invasif composé essentiellement d'histiocytes bien différenciés et de quelques lymphocytes et polynucléaires neutrophiles et éosinophiles[2]. L'indexmitotique est faible et les atypies cellulaires sont rares à absentes en accord avec un phénomène non tumoral[7], permettant notamment une différenciation avec une infiltration histiocytaire tumorale. Dans le cas présent, l'absence d'image histopathologique de malignité sur les prélèvements cutanés et épiscléraux, associée à des signes cliniques évocateurs et une réponse favorable initialement au traitement médical conduisent au diagnostic d'histiocytose réactionnelle systémique. Les marqueurs membranaires exprimés par les différentes cellules histiocytaires sont bien établis [2,11]. Bien que peu disponibles, une analyse immunohistochimique à la recherche de l'ensemble spécifique des marqueurs CD1+, CD4+, CD8+, CD11h+ et CD90 (Thy-1)+, CD11d- aurait permis d'appuyer le diagnostic. immunomodulateurs et immunosuppresseurs (prednisolone, azathioprine, cyclosporine, leflunomide, tétracycline/niacinamide)[7,10]. La réponse thérapeutique varie cependant selon l'individu et les molécules utilisées, nécessitant généralementun maintien du traitement sur le long terme. Parmi les molécules disponibles, les glucocorticoïdes apparaissent comme des 
leur efficacité et leur rapidité d'action[13]. Leur emploi est fréquemment rapporté dans des cas d'histiocytose réactionnelle mais avec des résultats

276

277

278

279

280

281

282

283

284

285

286

287

288

289

290

291

292

293

294

295

296

297

298 variables. Affolteret al. rapportent une réponse à des doses

immunosuppressives dans $50 \%$ des cas[11]. Paterson et al. décrivent également

une réponse variable avec un meilleur pronostic lors de prise en charge

précoce. Sur les 3 cas ayant répondu favorablement, tous présentaient des

signes de sclérite ou d'épisclérite et 2 d'entre eux présentait également des

nodules cutanés sans autre atteinte systémique[9]. Cependant, un

hypercorticisme iatrogène survient rapidement : signes cutanés, polyuro-

polydipsie, polyphagie et prédispositions aux ulcérations gastro-intestinales,

diabète, pancréatite, hépatopathie, troubles de la coagulation[14-16]. La

sévérité de ces effets secondaires ou, comme dans le cas présent, l'absence de

réponse au traitement conduit à les remplacer ou les associer à des

immunosuppresseurs de seconde ligne.

L'azathioprine est une thiopurine qui cible essentiellement l'immunité à méditation cellulaire [13]. Elle est fréquemment associée aux glucocorticoïdes dans la gestion d'affections auto immunes, pour leur effet synergique permettant de diminuer les doses utilisées et pour contrebalancer son délai d'action lent et inconstant, variant de quelques jours à plusieurs semaines[14].Dans le cas présent, une amélioration progressive des signes cliniques est notée suite à l'administration d'azathioprine. Cela corrobore les résultats de Palmeiroet al. rapportant3 cas d'histiocytose réactionnelle cutanée traités avec succès après la mise en place d'azathioprine. Ces résultats sont cependant inconstants : Peterson et al. décrivant un cas d'échec thérapeutique sur une histiocytose réactionnelle systémique traitée avec de la prednisolone et 
de l'azathioprine à dose immunosuppressive[9,10]. Les principaux effets secondaires attribuables à cette molécule sont une hépatotoxicité et une aplasie médullaire associées à d'éventuels troubles gastro-intestinaux[13,17]. L'étude récente de Wallischet al. décrit l'apparition d'une hépatotoxicité mixte (hépatocellulaire et cholestatique) dans $15 \%$ des cas dans un délai de 14 jours (13 - 22 jours), avec une augmentation des paramètres hépatiques d'un facteur 8 pour les PAL et 9 pour les ALAT. Les auteurs rapportent également asymptomatiques dans cette étude[17]. indiquée pour le traitement des maladies auto-immunes. Elle présente l'avantage d'être rapidement efficace avec peu d'effets secondaires (discret troubles gastro-intestinaux, rare hyperplasie gingivale ou surinfection opportuniste)[13,18]. Elle a été utilisée avec succès dans quelques cas isolés d'histiocytose réactionnelle cutanée et systémique[4,10]. D'autres molécules comme le leflunomide, ou l'association tétracycline - niacinamide sont également efficaces dans la gestion de cette affection[10,11,19].Dans le cas présent,le choix de la molécule immunomodulatrice s'est porté sur l'azathioprine en raison de l'efficacité décrite de cette molécule et pour des raisons de coût.

Malgré ces traitements, le pronostic reste réservé car l'évolution clinique de l'histiocytose réactionnelle est généralement longue, nécessitant un traitement immunosuppresseur prolongé et coûteux. Même si ces chiens décèdent rarement de cette affection à proprement parler, les traitements au long cours, 
leurs effets secondaires et la fréquence des récidives conduisent fréquemment à une décision d'euthanasie. Peu de données sur la survie de ces animaux sont disponibles. Paterson et al. (1995) rapportent 6 cas dont 4 avec des lésions de la sphère oculaire, parmi lesquels 3 sont euthanasiés et 2 cas sont en rémission après 6 et 18 mois de traitement respectivement. Gregory et al. (1998) rapportent 4 cas d'histiocytose réactionnelle systémique sans répercussion oculaire, dont 1 décède à 1 mois, et 3 sont toujours vivants après 18 mois de traitement.Pumphreyet al. (2012) décrit 1 cas d'atteinte rétro-orbitaire toujours vivant après 9 mois de prise en charge. Dans le cas présent, un décès brutal survient5 semaines après le diagnostic, sans que l'autopsie n'ait pu être réalisée. Une thrombo-embolie pulmonaire est principalement suspectée, favorisée par la pathologie immunitaire et le traitement corticoïde notamment. Une aggravation brutale de l'anémie, secondaire à des saignements digestifs notamment, reste possible. Enfin, l'évolution d'un sarcome histiocytaire, bien que non identifié lors du suivi, ne peut être définitivement écartée[1,6].

\section{$\underline{\text { Conclusion }}$}

Ce cas décrit la démarche diagnostique et thérapeutique d'une histiocytose réactionnelle chez un jeune Bouvier bernois et illustre les difficultés de prise en charge de cette affection. Malgré son caractère non tumoral et sa réponse théorique au traitement immunosuppresseur de par sa nature dysimmunitaire, le pronostic reste réservé du fait d'une réponse thérapeutique variable quel que soit le traitement immunosuppresseur instauré, de la nécessité d'un traitement onéreux au long cours et de la présence d'effets secondaires invalidants. Une prise en charge précoce semble cependant améliorer ce pronostic, notamment 

intégrée précocement dans le diagnostic différentiel des sclérites et épisclérites,particulièrementpour les races prédisposées.

\section{Conflit d'intérêt}

Aucun

\section{Références}

1. André C, Abadie J, Hedan B, et al. Proliférations histiocytaires de sarcome histiocytaire chez le Bouvier Bernois. Pratique médicale et chirurgicale de l'animal de compagnie $2010 ; 45: 9-17$.

2. Coomer AR, Liptak JM. Canine Histiocytic Diseases. Compendium on Continuing Education for the Practising Veterinarian2008;30 : 202 - 217.

3. Moore PF. Systemic Histiocytosis of Bernese Moutain Dogs. VeterinaryPathology $1984 ; 21$ : $554-563$.

4. Pumphrey SA, Pizzirani S, Pirie CG, et al. Reactive histiocytosis of the orbit and posterior segment in a dog. VeterinaryOphthalmology $2013 ; 16$ : $229-233$

5. Scherlie PH, Smedes SL, Feltz T, et al. Ocular manifestation of systemic histiocytosis in a dog. Journal of the American VeterinaryMedical Association $1992 ; 8: 1229$ - 1232.

6. Moore PF, Rosin A. Malignant Histiocytosis of Bernese Mountain Dogs. VeterinaryPathology $1986 ; 23: 1-10$.

7. Moore PF. A Review of Histiocytic Diseases of Dogs and Cats. 
VeterinaryPathology2014;51 : 167 - 184.

8. Padgett GA, Madewell BR, Keller ET, et al. Inheritance of histiocytosis in Bernese mountain dogs. Journal of Small Animal Practice $1995 ; 36$ : $93-98$.

9. Paterson S, Boydell P, Pike R. Systemic histiocytosis in the Bernese mountain dog. Journal of Small Animal Practice 1995 ; 36 : 233 - 236.

10. Palmeiro BS, Morris DO, Goldschmidt MH, et al. Cutaneous reactive histiocytosis in dogs: a retrospective evaluation of 32 cases.

VeterinaryDermatology $2007 ; \mathbf{1 8}: 332$ - 340.

11. Affolter VK, Moore PF. Canine cutaneous and systemic histiocytosis : reactive histiocytosis of dermal dendritic cells. American Journal of Dermatopathology $2000 ; 22: 40-48$.

12. Brearley MJ, Dunn KA, Smith KC, et al. Systemic histiocytosis in a Bernese mountain dog. Journal of Small Animal Practice 1994 ; 35 : 271 274.

13. Viviano KR. Update on Immunosuppressive Therapies for Dogs and Cats. Veterinary Clinics of North America: Small Animal Practice2013;43 : $1149-1170$.

14. Whitley NT, Day MJ. Immunomodulatory drugs and their application to the management of canine immune-mediated disease. Journal of Small Animal Practice2011;52 : 70 - 85 .

15. Behrend EN, Kemppainen RJ. Glucocorticoid therapy, Pharmacology, Indications, and Complications. VeterinaryClinics of North America:Small Animal Practice 1997; 27: 187 - 213.

16. Nichols R. Complications and concurrent disease associated with 

Animal Practice 1997; 27: 309 - 320.

401

402

403

404

405

406

407

408

409

410

411

412

413

414

415

416

417

418

419

420

421

422

423
17. Wallisch K, Trepanier LA. Incidence, Timing and Risk Factors of Azathioprine Hepatotoxicosis in Dogs. Journal of VeterinaryInternalMedicine $2015 ; 29: 513-518$.

18. Archer TM, Broothe DM, Langston VC, et al. Oral cyclosporine treatment in dogs: a review of the literature. Journal of VeterinaryInternalMedicine $2014 ; 28$ : 1 - 20.

19. Gregory CR, Steward A, Sturges B, et al. Leflunomide Effectively Treats Naturally Occuring Immune-Mediated and Inflammatory Diseases of Dogs That Are Unresponsive to Conventional Therapy. Transplantation Proceedings $1998 ; 30: 4143$ - 4148.

\section{Légendes}

Figure1 : Nodule cutané érythémateux sur le chanfrein après la tonte.

Figure2 : Nodules cutanés érythémateux sur le flanc droit après la tonte.

Figure3 : Aspect de l'œil gauche lors de la première consultation. Notez l'infiltration épisclérale, la congestion des vaisseaux épiscléraux et l'œdème cornéen ventral.

Figure 4 : Infiltrat histiocytaire périvasculaire formant des images en «bulbe d'oignon ».

Figure5 : Aspect de l'œil droit 24h après l'admission. Notez l'infiltrat limbique temporal, l'œdème cornéen et l'ulcère stromal.

Figure6 : Aspect de l'œil gauche 3 jours après la première consultation. Notez la progression de l'infiltration épisclérale et de l'œdème cornéen. 
Figure 7 : Aspect de l'œil droit 7 jours après la première consultation. Apparition d'une dyscorie dorsale et accentuation de l'œdème cornéen.

Figure 8: Aspect de l'œil droit après instauration de l'azathioprine. Notez la régression de l'œdème cornéen et l'infiltration épisclérale et limbique.

Figure 9 : Aspect de l'œil gauche après instauration de l'azathioprine. Notez la

429 régression de l'œdème cornéen et l'infiltration épisclérale.

Tableau 1 : Résultats de l'analyse biochimique et hématologique réalisée au cours du suivi à J+29. 



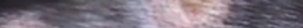

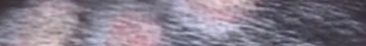

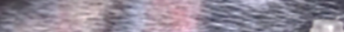

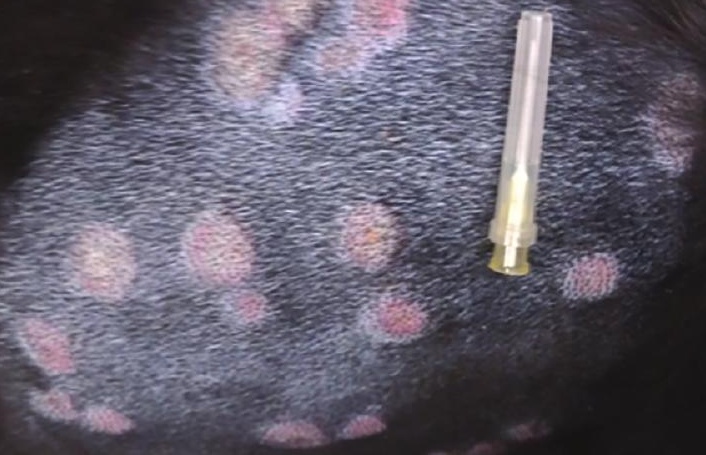

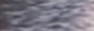

(t)

2its

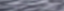

$x_{2}=2$

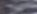

$15+2=0$

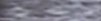

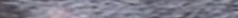

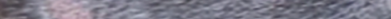

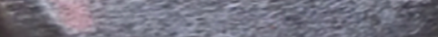




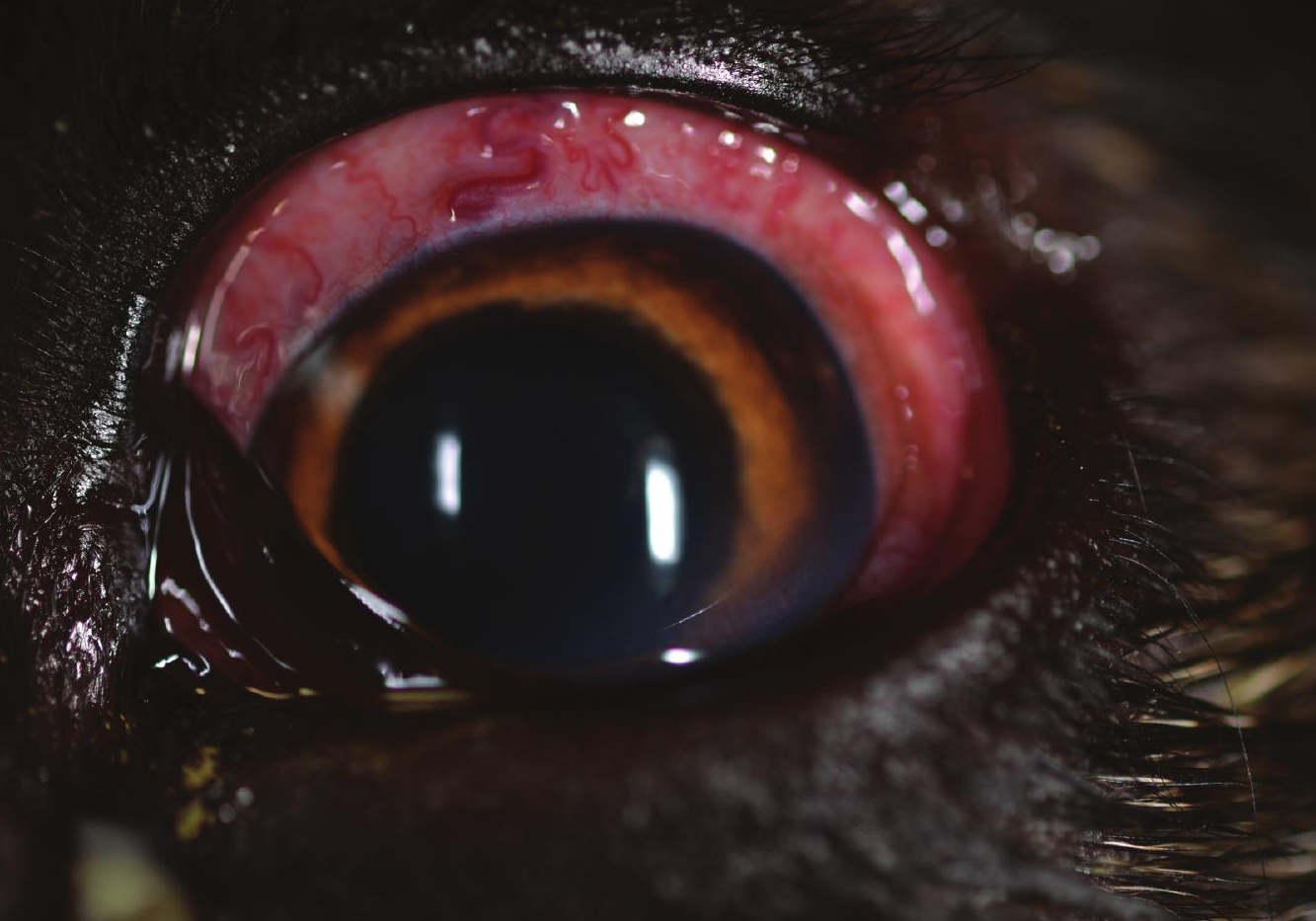




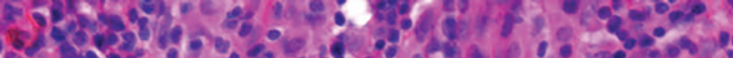

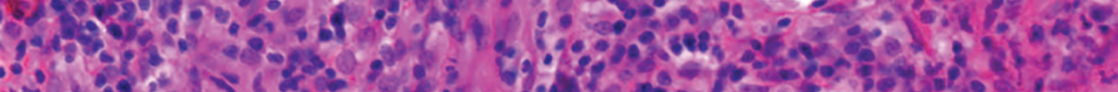
H.

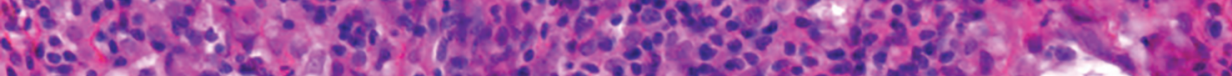

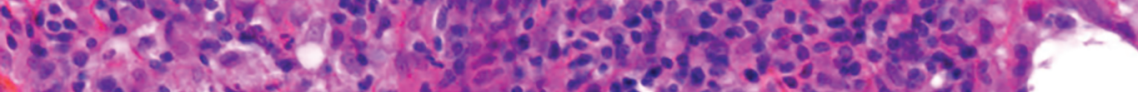

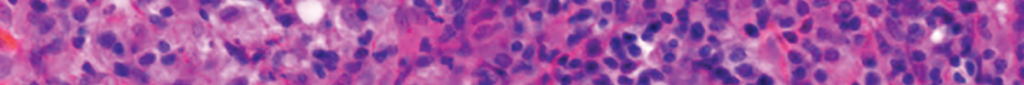

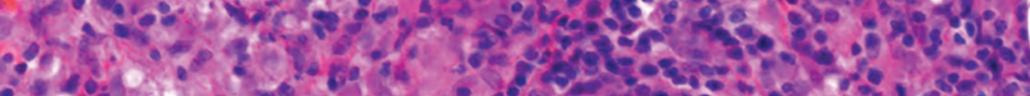

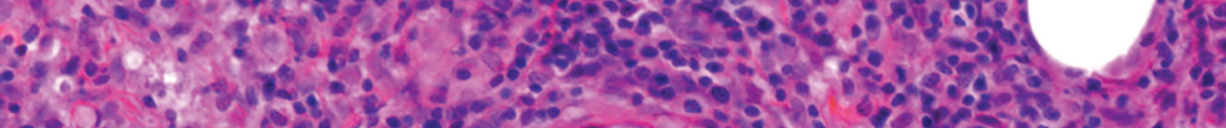
T.

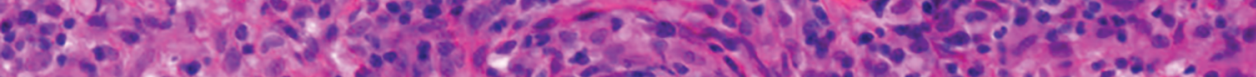

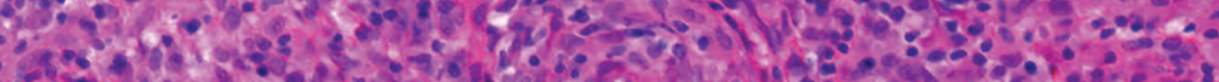

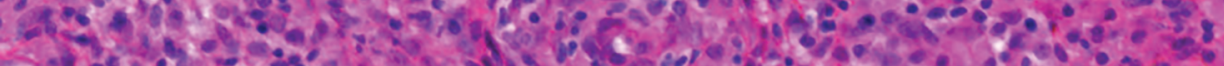

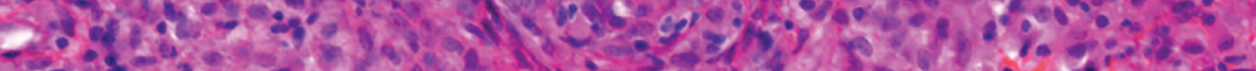

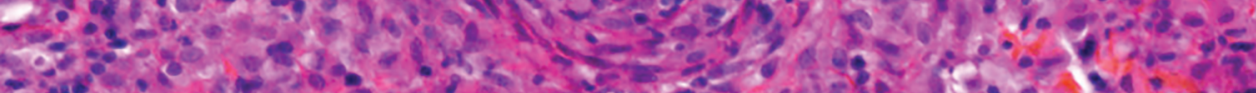

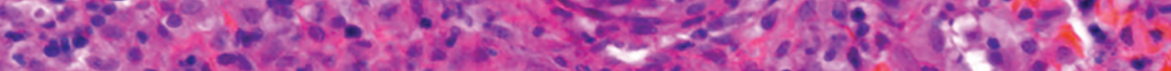

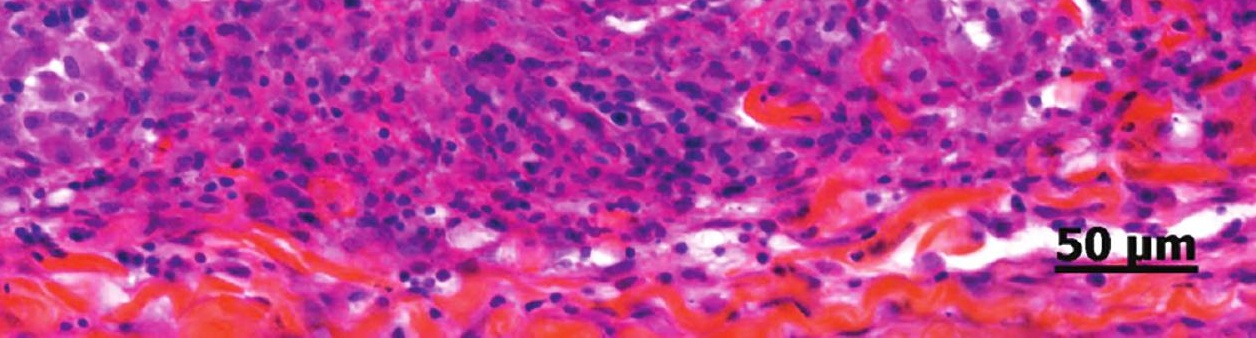




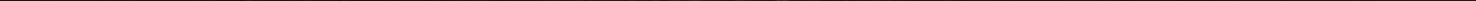




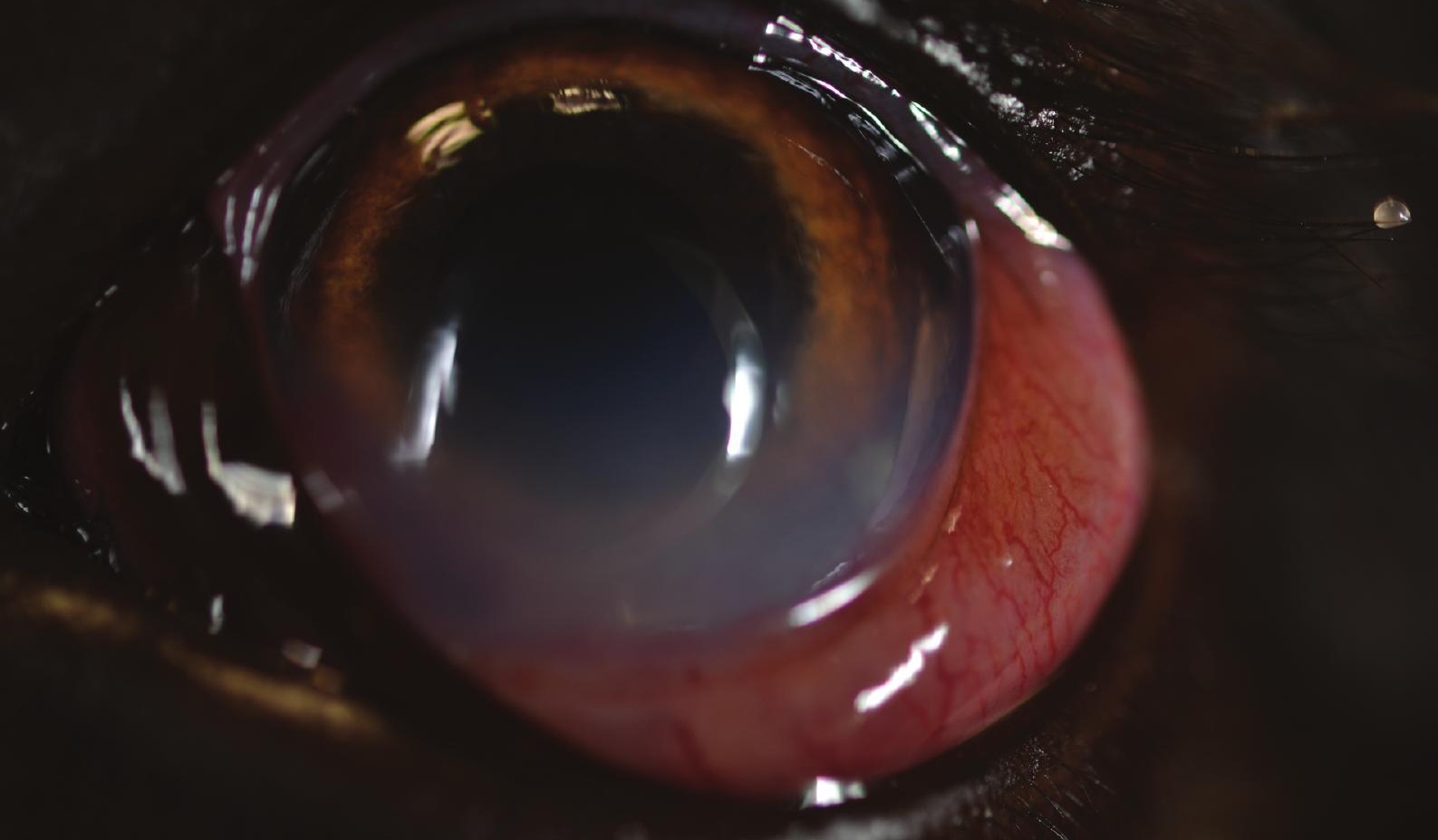




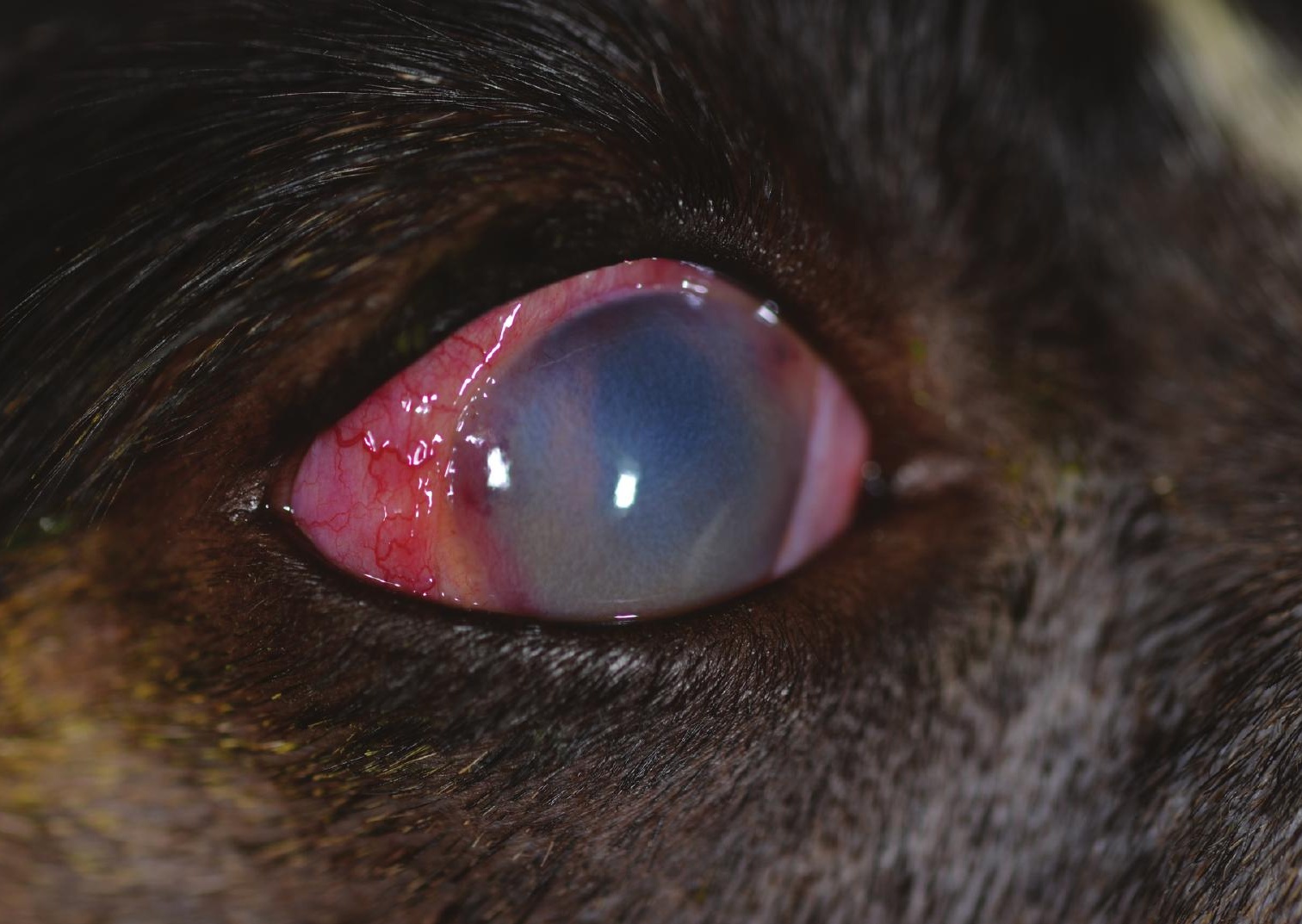




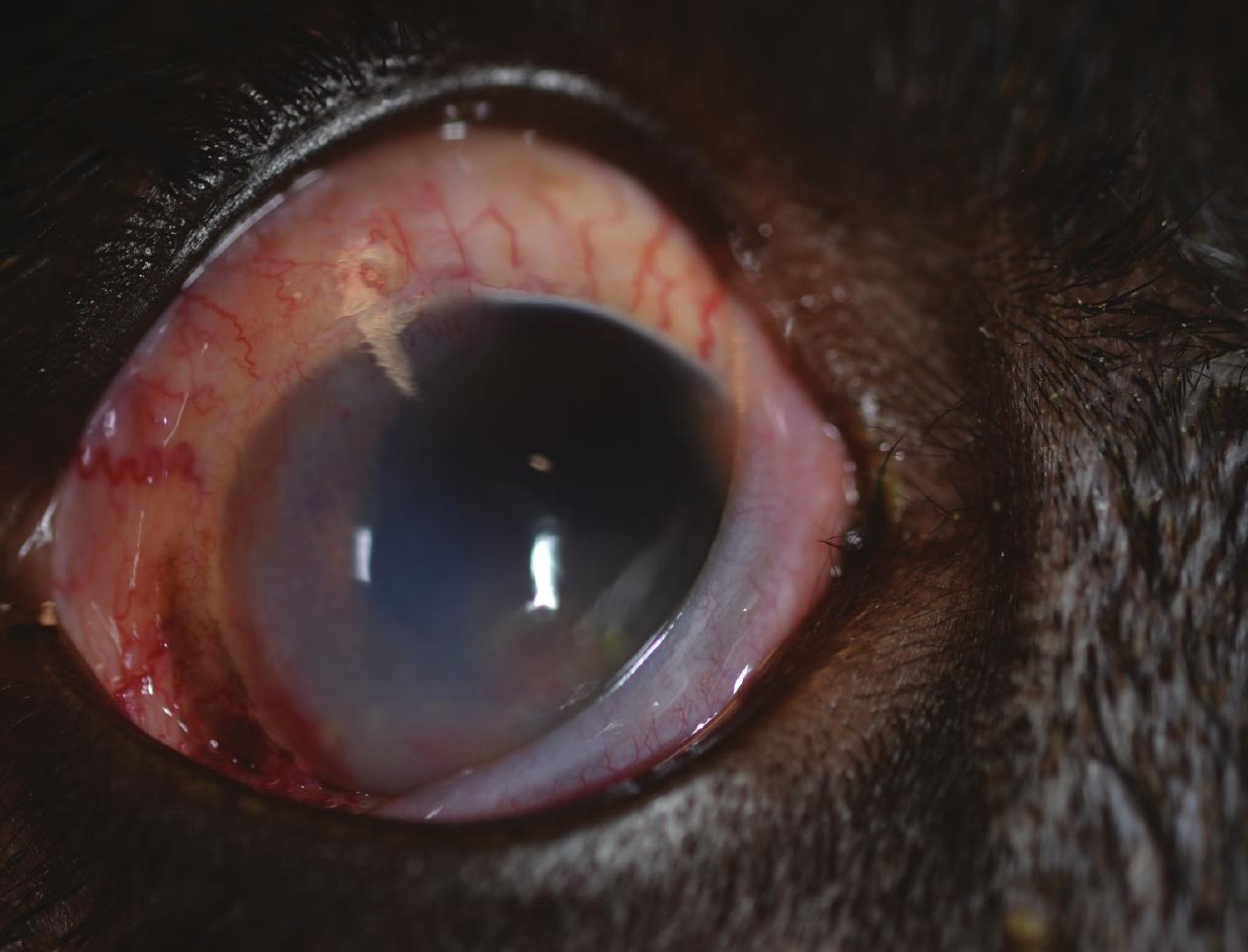



Valeurs Valeurs de référence

\begin{tabular}{|c|c|c|}
\hline$P A L$ & $6855 \mathrm{UI} / \mathrm{L}$ & $50-110 \mathrm{UI} / \mathrm{L}$ \\
\hline$A L A T$ & $1195 \mathrm{UI} / \mathrm{L}$ & $15-125 \mathrm{UI} / \mathrm{L}$ \\
\hline Hématies & 4,37 Millions/mm ${ }^{3}$ & $5,5-8,5$ Millions $/ \mathrm{mm}^{3}$ \\
\hline Hémoglobine & $10,2 \mathrm{~g} / 100 \mathrm{~mL}$ & $13,2-19 \mathrm{~g} / 100 \mathrm{~mL}$ \\
\hline Hématocrite & $30 \%$ & $40-55 \%$ \\
\hline$V G M$ & $69 \mu \mathrm{m}^{3}$ & $60-77 \mu \mathrm{m}^{3}$ \\
\hline TCMH & $23 \mathrm{pg}$ & $21-27 \mathrm{pg}$ \\
\hline CCMH & $34 \%$ & $32-36 \%$ \\
\hline Réticulocytes & $130,2 / \mathrm{mm}^{3}$ & $0-60000 / \mathrm{mm}^{3}$ \\
\hline Plaquettes & $498000 / \mathrm{mm}^{3}$ & $150000-500000 / \mathrm{mm}^{3}$ \\
\hline Leucocytes & $32500 / \mathrm{mm}^{3}$ & $6000-12000 / \mathrm{mm}^{3}$ \\
\hline Granulocytes neutrophiles & $27000 / \mathrm{mm}^{3}$ & $3000-9000 / \mathrm{mm}^{3}$ \\
\hline Granulocytes éosinophiles & $325 / \mathrm{mm}^{3}$ & $40-600 / \mathrm{mm}^{3}$ \\
\hline Granulocytes basophiles & $0 / \mathrm{mm}^{3}$ & $0-100 / \mathrm{mm}^{3}$ \\
\hline Lymphocytes & $1626 / \mathrm{mm}^{3}$ & $1000-3600 / \mathrm{mm}^{3}$ \\
\hline Monocytes & $3578 / \mathrm{mm}^{3}$ & $40-500 / \mathrm{mm}^{3}$ \\
\hline
\end{tabular}

Tableau 1 : Résultats de l'analyse biochimique et hématologique réalisée au cours du suivi à J+29. 